\title{
PRESTACIÓN DE SERVICIOS DE SALUD EN COLOMBIA: DILEMA ENTRE EL ESTADO Y EL MERCADO
}

\author{
Katherine Flórez Pinilla \\ Economista, Universidad Industrial de Santander UIS, Bucaramanga (Colombia). \\ E: mail: katheflorezpi nilla@gmail.com
}

\begin{abstract}
Resumen
La evolución de la organización de los servicios de salud en el mundo, permite deducir que ha estado influenciada por tendencias ideológicas y teorías económicas propias de la época que se debaten entre la conveniencia de instituciones como el Mercado y el Estado para garantizar el ejercicio de la prestación. Colombia no ha sido la excepción, se han evidenciado desde esquemas altamente centralizados hasta otros que buscan la formación de mercados. El actual esquema influido por corrientes neo-institucionalitas pretende ser un punto medio entre los extremos intervencionistas y la libre movilidad del mercado, en el cual el Estado tiene el rol de formulador de las reglas e incentivos que garanticen la competencia. Sin embargo, ninguno de los dos esquemas, ni su punto medio, han logrado la anhelada universalidad de la atención ni la equidad en el acceso.
\end{abstract}

\section{Palabras clave}

Regulación, Sistema de salud, Teoría económica, Estado, mercado.

\begin{abstract}
The evolution of health services organization on the world, let you deduce, this have been influenced by ideological tendences and economic theories typical for the age, which debate themselves between benefit of institutions like marketing or State Colombia has not been an exception and has shown sketches high-centralized and another ones looking for market's beginning. His current sketch, influenced by new institutionalist trends pretends to be neutral between interventionist extremes and the free mobility of marketing, in that, State acts as rules and awards maker for garanting birth of competitive markets. However, none of the two outlines, neither their half point, had achieved the so yearned protection in health for all, neither it has been able to put an end to the fragmentation of the services and of the population to consent to them.
\end{abstract}

\section{Key words}

Regulation, Health Services, Economic Theory, State, Markets. 


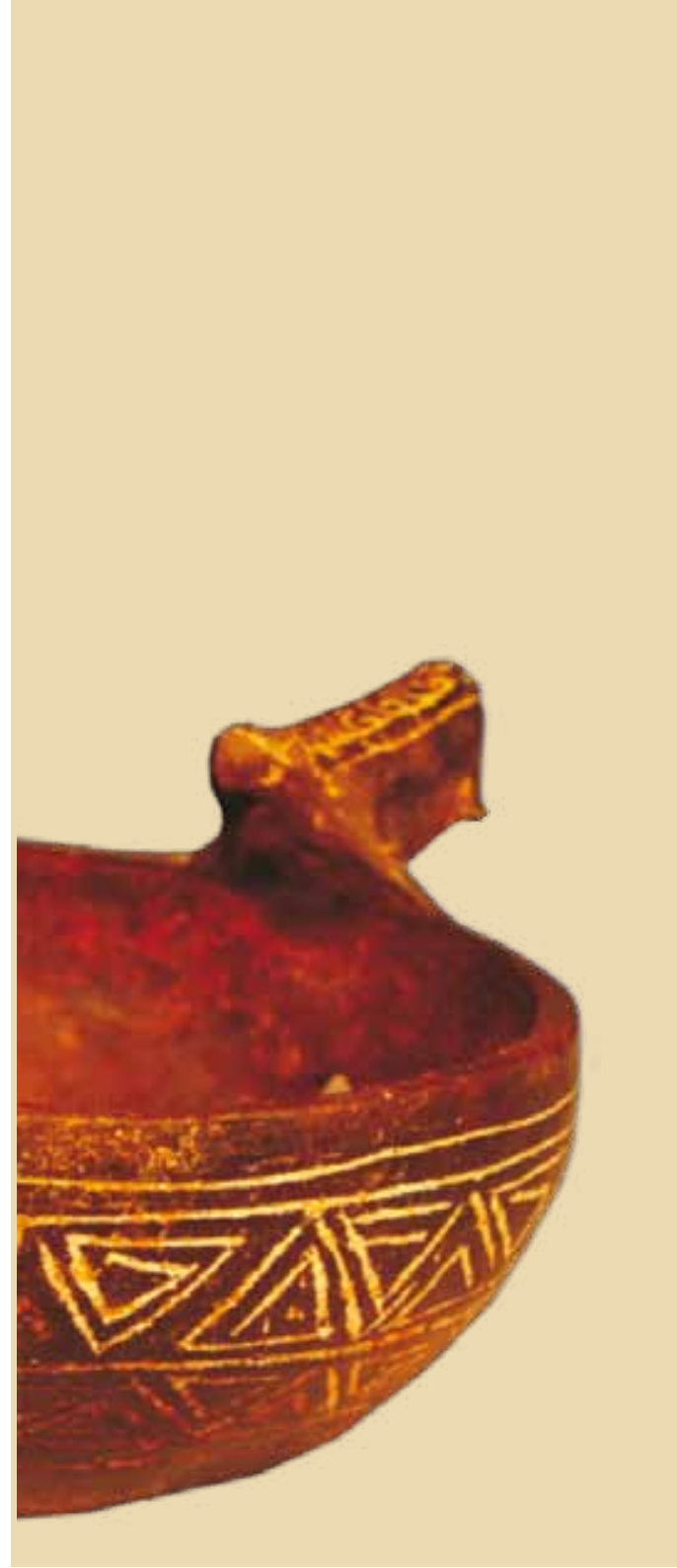

Detalle Figura

Cuenco doble 


\section{PRESTACIÓN DE SERVICIOS DE SALUD EN COLOMBIA: DILEMA ENTRE EL ESTADO Y EL MERCADO*}

Katherine Flórez Pinilla

La salud es la ausencia de la enfermedad, y por consiguiente de todas aquellas clases de dolor que son síntomas de enfermedad. Jeremy Bentham

La salud es una de las condiciones más importantes de la vida humana y un componente fundamental de las posibilidades humanas que tenemos motivos para valorar Amartya Sen

El debate sobre la conveniencia de la función regulatoria del Estado o del Mercado en la provisión de servicios de salud, adquiere relevancia en la actualidad dado el proceso de reformas que se viven actualmente en Latinoamérica. Muchos apoyan la premisa que el Estado debe brindar directamente todos los servicios, incluidos los de salud, que satisfagan un nivel básico de necesidades; otros, sin embargo, creen que las capacidades del Estado son limitadas, por lo cual sólo debe ocuparse de establecer las condiciones necesarias para que la misma sociedad se provea de manera libre todos aquellos bienes y servicios que necesita para existir.

* Este documento de reflexión se basa, en la ponencia presentada en el VI Concurso de Ponencias de Estudiantes de Economía “JESÚS ANTONIO BEJARANO”, en el marco del XXII Congreso Nacional de Estudiantes de Economía: "Economía del Siglo XXI: Desarrollo, problemática y nuevas perspectivas", realizado los días 2, 3, 4 y 5 de Octubre de 2007 Bucaramanga-Colombia. 
En la práctica los dos sistemas han mostrado distorsiones en su actuación, por un lado, los Estados poseen rasgos funcionales que no permiten que su capacidad sea ilimitada y, por el otro, el libre mercado ha demostrado a través del tiempo que no es oportuno en la prestación de servicios de salud. La inequidad en el acceso es un rasgo persistente que no se ha permitido abolir en ninguno de los dos esquemas.

El caso Colombiano no ha sido la excepción y desde hace una década se ha optado por un punto medio de organización: mecanismos estatales de regulación que posibiliten la simulación de un mercado de la salud. Aunque en teoría este punto medio es ideal para garantizar un comportamiento óptimo del sistema, la realidad muestra que el problema va mucho más allá de las formas de organización, los rezagos históricos en cobertura e inclusión así como la ineficiencia en la asignación de recursos son la principal barrera que limita los alcances del modelo.

A través del tiempo el sector sanitario ha estado influenciado por estructuras políticas, ideologías y tendencias conceptuales propias de la época. Particularmente en Colombia se puede decir que la planeación en salud ha seguido tanto líneas de acción y comportamientos político-administrativos del Estado como tendencias económicas mundiales: la acentuada centralización política-administrativa combinada con un alto proteccionismo característica de mediados de siglo XX, la descentralización de poderes y el liberalismo de los años noventa han marcado la trayectoria seguida para la prestación de los servicios de sanidad.

Este artículo tiene como propósito fundamental profundizar en la manera en que el Estado colombiano ha procurado brindar los servicios de salud a la comunidad y en los determinantes de esa función, así como sus principales errores y aciertos, todo esto enmarcado en el contexto de la teoría económica.

\section{SALUD: BIEN MERITORIO PARA LA PRODUCTIVIDAD}

La prestación de servicios de salud como organización social tiene su origen desde la Revolución Industrial, la cual, fundada en esquemas de propiedad privada condujo a que las personas sin bienes propios tuvieran, para poder sostenerse, que vender o alquilar su propia fuerza de trabajo. Ello supuso que la enfermedad, la vejez y el desempleo se convirtieran en riesgos importantes para la estabilidad económica del individuo. La enfermedad, la vejez y los períodos de inactividad siempre habían existido, pero la forma de producción de las sociedades preindustriales manejaba relativamente bien tales circunstancias: atendía a los enfermos en las familias o desplazaba a los viejos a tareas menos exigentes (Ortún, 1990, 35).

En respuesta a esta problemática $\mathrm{O}$. Bismarck en 1881, quien había observado que a los trabajadores les faltaban recursos para pagar servicios médicos que les permitiesen reducir su convalecencia y la ausencia en el trabajo, introduce el concepto de Seguridad Social ${ }^{1}$. Se promueve, desde entonces, todo un sistema de seguridad

1 Entendido como el conjunto de mecanismos de previsión de enfermedades y accidentes para los trabajadores. 
para los trabajadores fundamentado en las nociones de riesgo y contingencia, cálculo actuarial, obligatoriedad, solidaridad, protección a las necesidades sociales de los trabajadores de menores ingresos y un sistema de financiación calculado con base en la relación salarial entre trabajadores y empleadores (Cañón Ortegón, 1988, citado por OPS, 2002).

Se puede afirmar entonces que en el momento en que se transforman las relaciones de producción en la economía, el estado de salud de las personas adquiere importancia y su atención se extiende y se profesionaliza para atender las necesidades. Desde entonces se tratan de articular esfuerzos para que esta atención se extienda de manera gradual a toda la población lo cual supone un problema importante para la sociedad.

En un primer momento la prestación de servicios de salud se enmarcó por la ideología económica liberal que defendía el mercado como la mejor forma de asignar los recursos y organizar la sociedad. De acuerdo a su capacidad de pago y preferencias, los individuos adquirían servicios de sanidad. No obstante, tal argumento se derrumbó con la crisis de 1929, lo cual fortaleció los poderes del Estado como organizador y protector de la sociedad.

Más adelante, el periodo de la posguerra supuso un fuerte intervencionismo estatal en el cual cobraría importancia el concepto de equidad para garantizar así un nuevo conjunto de derechos sociales como los derechos a la educación, a la protección de la salud y a un entorno correcto (Navarro, 1984, citado por Ortún). La garantía del derecho a la salud se instrumentó mediante diversas formas de planificación de los países que al final configuraron lo que hoy conocemos como sistemas de salud.

El comienzo del Estado de Bienestar y de la intervención en el sector salud se hizo evidente con crecientes presupuestos e inversiones estatales en infraestructura hospitalaria; con los principios del asistencialismo curativo. Sin embargo, fallas estructurales del Estado y circunstancias adversas en la economía mundial llevaron a cambiar tal esquema benefactor por uno totalmente opuesto a final de siglo.

La panorámica general muestra que tanto el Estado como el Mercado han adquirido protagonismo como prestadores de atención en salud. Si bien diversos enfoques de la economía pueden brindar luces sobre formas, alcances y limitaciones que posee cada esquema, una perspectiva histórica puede describir sus resultados y comportamiento en las sociedades.

\section{PROBLEMA TEÓRICO: DILEMA ENTRE EL ESTADO Y EL MERCADO}

Un primer enfoque teórico argumenta que la salud se comporta como cualquier bien de mercado, donde los consumidores que buscan maximizar su utilidad deciden qué comprar de acuerdo a su restricción presupuestaria. El consumidor al pagar directamente puede acceder a los servicios de salud al costo más bajo, dado un 
ambiente competitivo entre proveedores privados. Todo esto se valida con el concepto de eficiencia el cual hace alusión al nivel de producción de bienes y servicios que se obtienen al menor costo y son distribuidos de manera socialmente óptima.

Sin embargo, este modelo no ha sido compatible con la realidad pues para que una economía de mercado alcance un óptimo en el sentido de Pareto o maximice el bienestar social debe cumplir supuestos irreales como: información perfecta, racionalidad individual; cero externalidades y competencia perfecta en los mercados, los cuales no se evidencian en ningún mercado y producen distorsiones, ésta es una de las primeras razones que incentivan la intervención estatal.

Kenneth Arrow en 1963 argumentó las características económicas intrínsecas a la atención sanitaria, así como también aquellas que se derivan de la intervención política a las características intrínsecas. Constituyen características intrínsecas de los servicios sanitarios que evidencian fallos de mercado: la existencia de bienes públicos, externalidades, incertidumbre y asimetrías en la información.

\section{Bienes públicos}

Aunque la asistencia sanitaria no se concibe como bien público en el sentido económico, algunos servicios directamente relacionados con la salud si lo son, por ejemplo, servicios de higiene alimentaria y de cuidado del ambiente. La respuesta política a esta problemática ha sido la provisión totalmente pública de estos servicios.

\section{Externalidades}

Enfáticamente la prestación de servicios de salud se caracteriza por la presencia de externalidades; una persona enferma puede trasmitir su dolencia a otras más, y así otras. Igualmente varios factores en el ambiente influyen directamente en el estado de la salud, aquí cobra importancia los programas de saneamiento básico y de prevención para grandes grupos poblacionales.

\section{Incertidumbre}

La respuesta institucional a la presencia de incertidumbre entre los consumidores sobre la presencia de enfermedad y, por tanto, incertidumbre en la utilización de servicios de salud ha consistido en el establecimiento de mecanismos de seguros tanto estatales como privados. Sin embargo, el efecto secundario más importante del seguro es el consumo excesivo o riesgo moral, el cual implica que cuando el consumidor, enfrentado a un precio cercano a cero en el momento el consumo, demanda servicios cuya valoración marginal está por debajo de su coste marginal social. Otros efectos de los seguros son la rigidez de precios, la pérdida de economías de escala, información insuficiente para el establecimiento de primas y selección adversa o propensión de los individuos con alto riesgo de enfermar a comprar seguros 
$\mathrm{y}$, al mismo tiempo, la tendencia de los aseguradores a asegurar a los individuos con bajo riesgo de enfermar denominada también selección de riesgos.

\section{Asimetría de información}

Uno de los principales problemas que origina la presencia de asimetría de información es la demanda inducida, que se refiere a aquella parte de la demanda iniciada por el proveedor que está por encima de lo que el paciente hubiera estado dispuesto a pagar en el supuesto de que éste tuviera plena información o, como mínimo, la misma información que el médico. Es decir, la diferencia que existe entre la información que está disponible para los enfermos y la que poseen sus médicos lleva a un fuerte dominio profesional de los prestadores de los servicios en las decisiones, lo cual hace posible que los médicos y los hospitales induzcan la demanda y fijen precios que no obedecen a las leyes del mercado.

El establecimiento de una relación de agencia ha sido la respuesta institucional a tal asimetría en la información, en dónde el paciente (principal) delega en el médico (agente) la toma de decisiones en nombre del primero. Cuando el médico realiza tal toma de decisiones únicamente con las variables que afectan al paciente, la relación de agencia es completa. Si el agente, de forma consciente o inconsciente incorpora variables que le son relevantes a él pero no al paciente, existe a una relación de agencia imperfecta.

Las repuestas sanitarias al fenómeno de la demanda inducida han consistido en confiar más en la regulación de oferta que en la regulación de la demanda como instrumento de política sanitaria, y desconfiar de las frecuentaciones como criterios de planificación, ya que éstas vienen explicadas por la oferta existente.

La demanda inducida por el proveedor tiene asimismo dos importantes implicaciones económicas, una de tipo positivo y otra de carácter normativo. Positivamente, la oferta y la demanda dejan de ser independientes y se incumple una de las condiciones para que el mercado sea un mecanismo eficiente de asignación de recursos en el sector sanitario. Normativamente, la soberanía del consumidor deja de ser la justificación de las pautas de utilización (Ortún, 1990, 31). Además, según Chicaiza (2002), cuando se necesita atención médica, las restricciones económicas de precios, riqueza y el presupuesto no son una barrera tan efectiva como en el consumo de otros bienes.

Estas características de los servicios de salud proporcionan una importante justificación teórica para intervenir en el mercado. Una posible solución que se propone es crear un sistema sanitario público con racionamiento de la oferta hasta el nivel de asistencia eficiente. Ello requiere cambios organizativos e incentivos para introducir las consideraciones de eficiencia en la práctica clínica. También puede pensarse en mejorar la información al consumidor y propiciar una elección económicamente consciente de éste. 
En respuesta a las fallas de los mecanismos del mercado, otros enfoques como el de Bienestar Ampliado (extra-welfarism) propuesto por Sen (1979), Williams (1972) y Culyer (1989) establece que existe información adicional sobre el bienestar no derivado de las mercancías, como determinadas características de los individuos: como la de si son felices, están libres de molestias, pueden escoger, tienen movilidad física y si son honestos, entre otras. Esta concepción trasciende a la Economía del Bienestar tradicional: no excluye la información acerca de la satisfacción derivada del consumo, pero la suplementa con otros aspectos de los individuos que incluye también cómo se relacionan entre sí y cómo se relacionan grupos y clases sociales (Culyer, 1989 citado por Ortún).

En economía de la salud, este enfoque adopta la salud como un "bien protector" el cual se debe maximizar, pues se considera producto de los servicios sanitarios a los que califica de productos intermedios. El producto final será la consecución o recuperación de un nivel de salud que sin esos productos intermedios no se hubiera conseguido. Aparte de los fallos del mercado reseñados, la visión del Bienestar Ampliado permite que tanto los juicios de valor sobre las cuestiones distributivas como la calificación de ciertos servicios como dignos de tener su consumo protegido constituyan razones adicionales para la intervención del Estado en el sector sanitario.

\section{PAPEL DEL ESTADO}

Alternativamente al enfoque dominante del mercado se propone entonces un papel activo del Estado en la toma de decisiones del sector salud. Entre las funciones interventoras que ha ejercido el Estado en el sector salud se encuentran principalmente: la financiación, la producción, la provisión y la regulación.

De acuerdo a Ortún, una financiación pública elevada tiene a priori cuatro ventajas: permite un mejor control del gasto total, facilita la orientación de recursos hacia donde pueden ofrecer un mayor producto en términos de salud, responde a la creciente preocupación de las sociedades por la equidad, además tiene mayor legitimidad democrática asignar recursos a la salud según necesidad que según capacidad de pago.

La producción comprende todas aquellas organizaciones que producen los insumos necesarios para los servicios (universidades, centros de investigación y complejo médico-industrial) también admite distintas combinaciones públicoprivadas, todo depende de la solvencia y la capacidad estatal como productor directo. Independientemente de la posición teórica al respecto, se debe optar por la combinación público-privado que más contribuya al bienestar de la sociedad de acuerdo a las capacidades de la misma. La combinación de estos insumos en un proceso de producción y en una estructura organizacional particular determina la prestación de una serie de productos (servicios de salud) que generan un resultado (cambios en el estado de salud del usuario). 
Además de las funciones anteriores, el Estado se ha encargado también de regular numerosos aspectos del sector al establecer, implantar, y monitorear normas para la protección de los ciudadanos en los ámbitos que afectan en alguna medida el estado de salud. Por tanto, ha sido encargado de establecer las reglas del juego así como de brindar dirección estratégica al sistema en su conjunto.

A pesar del alcance de estas funciones, el Estado posee grandes falencias que impiden un óptimo desempeño del sistema, las más evidentes y frecuentes son: predominio de grupos de interés en la asignación de recursos; corrupción; falta de incentivos para innovar y promover la eficiencia; ausencia de sensibilidad a las necesidades del usuario y desigualdades originadas por el otorgamiento de poderes y privilegios que propician la falta de equidad y la corrupción (Restrepo, 2000; 669).

\section{HACIA UN MERCADO DE SALUD CORREGIDO}

A finales de siglo XX surge una teoría que pretende responder a los fallos de mercado e impedir la intervención directa del Estado. Su precursor, Alain Enthoven (1993), la denomina como competencia manejada (Managed competition) y la define como una "estrategia de compra de servicios para obtener el máximo valor por el dinero que pagan los consumidores". Este modelo constituye un híbrido entre la regulación estatal y la competencia que pretende ofrecer servicios de salud individuales a través de un mercado corregido por medio de reglas de juego claras y relaciones de agencia establecidas. Se basa en el establecimiento de una serie de reglas que estructuran la competencia y premian con más clientes e ingresos a aquellos planes de salud que más satisfagan a los usuarios en calidad, costos y atención (Enthoven; 1993, 6).

Por un lado, se introduce la figura de un Patrocinador (sponsors) el cual se encarga de garantizar ciertas condiciones: protección financiera para todos, esquemas de organización de servicios que informen a los usuarios de los costos para ellos elegir la entidad que les proveerá los servicios. Además, diseñar y administrar las reglas para la competencia que no compensen a los planes de salud que seleccionan el riesgo, que segmenten el mercado, o que de alguna manera incumplan las reglas de juego.

La teoría supone que los patrocinadores son compradores de servicios racionales y activos que contratan los planes de salud en nombre de un grupo grande de afiliados y que, en forma permanente, observan, estructuran y ajustan el mercado, tendiente por naturaleza a evitar la competencia por precio.

Los patrocinadores se pueden presentar bajo la forma de entidad gubernamental, organización, sindicato o empresa. Su función es contratar con un asegurador de salud los beneficios cubiertos, los precios, los procedimientos de afiliación, y otras condiciones para la participación de los usuarios. Este agente estructura y ajusta el mercado de proveedores para establecer reglas de equidad, crear una demanda elástica al precio, y evitar una selección de riesgo. La principal motivación del 
patrocinador es conseguir servicios de salud de alta calidad para sus beneficiarios con los recursos disponibles.

El modelo reconoce las fallas existentes en el mercado y dispone de ciertas herramientas que pretenden eliminarlas (Enthoven, 1988, en: Retrepo, 2000):

1. Determinación de precios: determinar las primas por riesgo comunitario, ajustar los riesgos o mecanismos de reaseguro que garanticen que el proveedor no pierda y, por tanto, no practique selección adversa.

2. Estandarización de los planes de beneficios: al homologar los beneficios ofrecidos por todos los seguros de salud, de modo que sean comparables para evitar la segmentación de mercados, la selección de riesgos y la diferenciación de productos.

3. Proceso periódico de afiliación: son periodos abiertos de afiliación en los cuales los usuarios pueden afiliarse o desafiliarse y cambiar de asegurador. Esta modalidad evita la selección adversa y entrega a los usuarios la información necesaria para realizar una elección adecuada, así como para abandonar planes de salud que no los satisfagan.

4. Continuidad en la cobertura: el patrocinador debe garantizarla mediante contratos con los aseguradores afiliación permanente.

5. Vigilancia de los planes: los patrocinadores deben observar el desempeño de cada seguro, vigilar signos de selección de riesgos, e incluir mecanismos que prevengan tal fenómeno en los respectivos contratos.

6. Garantía de Calidad: realizar seguimiento a la calidad de los servicios.

7. Incentivar la competencia: los patrocinadores deben promover la entrada de nuevos competidores al mercado.

8. Manejo de subsidios: manejar subsidios cruzados y subsidios para los más pobres, los cuales deben exceder el precio de la prima más barata.

Este esquema pretende formar un mercado corregido al eliminar las fuentes de fallas de mercados: free riders, segmentación de mercados y selección de riesgos. El patrocinador tiene la pretensión de perfeccionar el mercado con el objetivo final de garantizar un cuidado de alta calidad a un costo muy bajo, más organizado y entregado de una manera más coherente y satisfactoria.

\section{EVOLUCIÓN DE LA REGULACIÓN Y LA PRESTACIÓN DE SERVICIOS EN COLOMBIA}

Desde los inicios de la industrialización en Colombia se evidenció, según Hernández (1999), una fragmentación en la prestación de servicios de salud justificada por los conceptos de propiedad y caridad. Para el periodo de 1880-1936 existía atención 
en salud privada para ricos según capacidad de pago y salud para pobres a cargo de la Iglesia u organizaciones de beneficencia. Todo esto dentro de un esquema en donde el Estado se mantenía al margen y no actuaba por las necesidades sociales de salud de gran parte de la población. No obstante, la incursión de la economía agro-exportadora nacional en el mercado mundial establecería nuevas demandas y exigencias a la población para fortalecer la capacidad productiva del país ante el resto del mundo, lo cual obligaría al Estado a revaluar sus políticas de bienestar.

Se empezaron a realizar esfuerzos desde las empresas y en menor medida desde el Estado para garantizar higiene y sanidad en las actividades productivas; especialmente en las zonas estratégicas para la economía, ejemplo de esto son los puertos que permitían el transporte de los productos como el banano y el café, al comercio internacional. Debido a que la higiene era una condición básica de las compañías norteamericanas exigían para el ingreso de capitales extranjeros en el país (Hernández, 1999).

Sin embargo, epidemias afectaron grandes masas de trabajadores lo cual tuvo un impacto negativo en la productividad nacional, situación que condujo a pensar en un modo en que se impactara positivamente en la salud de aquella parte de la población vulnerable y especialmente en la clase trabajadora. Los esfuerzos por parte del gobierno no fueron muchos pero, por primera vez, se estableció una transferencia de recursos estatales hacia las organizaciones de beneficencia existentes para otorgar atención individual a los pobres.

Ante la presencia de enfermedad las personas que carecían de recursos recurrían a prácticas médicas populares no científicas, producto de creencias culturales, ubicadas en un lugar marginal y subvalorado por los servicios legalmente aceptados (Hernández, 2000). Toda esta situación demuestra que los servicios de salud constituían una organización social fragmentada de acuerdo a la capacidad de pago de las personas, quienes la tenían recurrían al mercado y quienes no solicitaban atención en los establecimientos de caridad.

Después del colapso del sistema de libre mercado y de las doctrinas económicas clásicas, se instaura la propuesta intervencionista de Keynes como la mejor alternativa para garantizar estabilidad económica y social. En el país se evidencia esta tendencia desde finales de los años treinta e inicios de los cuarenta, la cual incide también en la forma de proveer atención en salud.

Como primeramedida de intervención se instauran, desdelos cuarenta, mecanismos de seguro de enfermedad para los trabajadores formales (Seguro Social), basados en el modelo alemán propuesto por Bismarck de seguros sociales obligatorios, pero su desarrollo era precario todavía. Existía pluralidad de regímenes, organizaciones y sistemas de financiación así como diversas técnicas de administración financiera de los riesgos cubiertos (OPS, 2002). Este seguro tenía un carácter personalizado del aseguramiento de los riesgos, contingencias de enfermedad y accidentes, muy pocas organizaciones aseguradoras promovían servicios preventivos. Esta tarea la

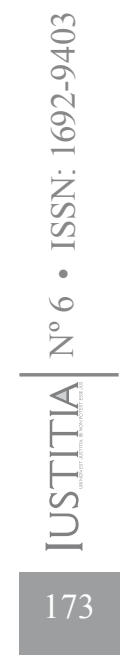


garantizaba el Estado en conjunto con otras organizaciones, no existía ningún tipo de integración y coordinación entre sí, tampoco una igualdad en la calidad de los servicios. Los sectores deprimidos de la sociedad dependían de los organismos de beneficencia.

Varios cambios en este esquema se establecerían después de la segunda guerra mundial, periodo en el cual se establecen una serie de derechos sociales de acuerdo a las necesidades de la población y al creciente movimiento sindical y socialista que atacaba las estructuras políticas capitalistas. Entre la formulación de estos derechos se destaca la salud por estar relacionada íntimamente con la vida y la integridad física, lo cual acentúa las tendencias proteccionistas y se adoptan diversas estrategias a nivel mundial para garantizar tales derechos. La salud sería asumida como una inversión pública y los Estados deberían unir esfuerzos para consolidar Sistemas de Salud regulados a través del aseguramiento universal o de un servicio único de salud administrado y prestado directa o principalmente por el Estado.

En los años sesenta, en el marco del Estado de Bienestar y de centralización de los poderes políticos y administrativos, se establecen iniciativas de planificación para consolidar los servicios de salud como un Sistema único integrado. Pero tal propósito, según Hernández (2000), fue influido por la lógica de los partidos políticos predominantes lo cual obstaculizó, en cierta medida, el desarrollo de mecanismos eficaces que integraran la prestación de servicios y garantizarán la cobertura a toda la población.

Sólo hasta la década de los setenta se logra consolidar un sistema de atención, a saber: Sistema Nacional de $\mathrm{Salud}^{2}$ afianzado por la nueva orientación estatal en los servicios públicos y la influencia de organizaciones internacionales como el Banco Mundial y la Organización Mundial de la Salud. Éste se constituiría por un conjunto de organismos, instituciones, agencias y entidades, existentes y nuevas, que tenían como objetivo procurar la salud de la comunidad, en los aspectos de promoción, recuperación y rehabilitación. El objetivo de este sistema era articular las diferentes instancias de prestación de servicios de salud, la privada personas con capacidad de pago, la pública para población vulnerable y la de seguridad social para los trabajadores.

En el entorno oficial la organización estaba liderada por el Ministerio de Salud ${ }^{3}$, quien se encargaba de controlar Servicios Seccionales de Salud de los departamentos, que a su vez controlaban la red hospitalaria de cada territorio ${ }^{4}$. La financiación se realizaba con recursos fiscales que se transferían a los departamentos, para que cumplieran funciones de diagnóstico, planificación, administración, inspección de recursos y vigilancia epidemiológica; $y$, además, financiara el funcionamiento 
de los hospitales de su jurisdicción de acuerdo a una metodología de presupuesto histórico ${ }^{5}$. Esta modalidad de asignación de recursos hacia los hospitales incentivaba la ineficiencia pues en la medida en que los presupuestos hospitalarios crecían se incrementaba el presupuesto de la siguiente vigencia. Evidentemente constituía un incentivo perverso que iba a degenerar en corrupción. De acuerdo a Orozco (2005), la red hospitalaria pública tenía dos razones más para hacerse, cada vez, menos eficiente: el exceso de carga prestacional hasta niveles insostenibles y los nombramientos innecesarios de trabajadores

Esta multiplicidad de funciones a cargo de las seccionales constituyó un esquema ineficiente, burocratizado y propenso a la corrupción. Se puede decir que aunque fue el período floreciente de la inversión estatal en infraestructura de hospitales públicos de segundo y de tercer nivel de complejidad con excelente dotación tecnológica, esta inversión no brindó los resultados esperados de cobertura y calidad en la prestación y, además, no tuvo un impacto en la salud pública evidenciándose un total abandono de los servicios de primer nivel de complejidad.

Según destacan estudiosos del tema, Sánchez (1990), Orozco (2005) y Hernández (2000), el Sistema Nacional de Salud aunque se lo propuso no logró articular los diferentes sub-sectores de la prestación de servicios de salud que existían, sino que acentuó las diferencias entre ellos. Además, se hizo evidente que las políticas no podían seguir planificándose centralmente, resultaba necesario actuar de acuerdo a las situaciones particulares de cada territorio y de las comunidades y permitir la articulación de los individuos en las políticas.

A finales de los ochenta se empieza a ver la necesidad de separar las funciones de salud que ejercían los Servicios Seccionales y otorgarle responsabilidades a los municipios de planificar la salud. Hechos como el fortalecimiento de las finanzas regionales y locales en 1983, y la elección popular de alcaldes en 1986, dan inicio al proceso de descentralización que se reforzaría totalmente con la Constitución de 1991. Al igual que otros sectores, la salud inició su proceso de reformas conjuntamente con el proceso de modernización del Estado para la estabilización después de la crisis del intervencionismo en los países de la periferia.

La más importante de las reformas comienza con la puesta en marcha de la Ley 10 de 1990 que prioriza la descentralización del sistema de salud y constituye "Sistemas Locales de Salud". Con esta creación los municipios podrían acceder a los recursos del situado fiscal de salud para las atenciones de menor complejidad. Para esto los municipios debían crear un Fondo Local de Salud, diseñar un sistema de información en salud que les permitiera mantener un diagnóstico actualizado de las condiciones de salud de la población y la elaboración de un Plan Local de Salud que permitiera controlar a través de indicadores la ejecución de los recursos de salud.

5 El cálculo del valor transferido a la institución dependía de cuánto había gastado el año anterior en recurso humano, gastos generales, mantenimiento de infraestructura física e insumos hospitalarios. 
Con la nueva Constitución se refuerzan las funciones descentralizadoras en el sector, pero el cambio más importante fue la asignación de nuevas responsabilidades a cargo del Estado para proporcionar servicios de salud a la población. Si bien, antes el ente mayor se encargaba directamente de la provisión de servicios dotado de una enorme infraestructura para proporcionarlos de manera gratuita, ahora se establecía el papel de garante de la prestación universal de los servicios y de establecer las reglas del juego que permitieran la convergencia de todos los actores prestadores y de toda la población de manera igualitaria en un solo sistema. Para esto se diseñó un nuevo esquema de regulación de los servicios y la atención en salud conocido como Sistema de Seguridad Social en Salud.

Es de destacar que todo lo plasmado en la carta constitucional va acorde con el enfoque de garantía de los derechos de los ciudadanos, resultado de un proceso de conciliación política, en donde se presenta la salud como un derecho prestacional ${ }^{6}$ lo cual lo hace exigible y defendible por los colombianos, aunque no pertenezca al rubro de derechos fundamentales.

La Constitución señala que el Estado debe cumplir las funciones de dirección, coordinación y control de los servicios de Seguridad Social ${ }^{7}$ según los principios de eficiencia, universalidad y solidaridad ${ }^{8}$. En este sentido, el principio de la eficiencia pretende garantizar, dado que los recursos son escasos, la mejor utilización de recursos en el sistema que resulte en el mejoramiento del estado de la salud de la población. Para este propósito el Estado formuló priorizar y focalizar la inversión en salud en aquellas atenciones de alta externalidad positiva, es decir, en las que conlleven a una mejor relación costo beneficio que irradien un mejoramiento de la salud colectiva.

La universalidad se refiere al acceso de la totalidad de la población a un paquete básico de atención en salud. Sin embargo, para aquella parte de la población que se encuentra en condiciones de vulnerabilidad económica el Estado, en su papel de garante del acceso, crea una política de subsidios a la demanda. No obstante, bajo el enunciado de solidaridad, se establece que las personas con poder adquisitivo deben compartir con la población sin capacidad de pago sus recursos y que las personas que gocen de buena salud deben compartir con los enfermos, para esto se establecen dos mecanismos: seguro de salud de carácter universal y subsidios cruzados.

Este nuevo sistema se instituye por medio de la Ley 100 de 1993, la cual al seguir los principios constitucionales trae consigo una forma diferente de concebir el sistema de salud no como un conjunto de organizaciones que prestan servicios, sino uno que pretende responder de manera organizada a las necesidades de la población

6 Constitución de 1991, Capítulo II: derechos sociales, económicos y culturales.

7 En Colombia la Carta Constitucional define la seguridad social como el acceso a servicios de protección y salud y pensiones.

8 Véase Artículo 48, Constitución de 1991. 
con un esquema extensivo de aseguramiento. Se concibe este esquema de seguro como un pluralismo entre instituciones públicas y privadas, y entre financiamiento público y privado que logra integrarse e incluir a toda la población clasificándola por su capacidad de pago.

Se crea por Ley un régimen contributivo para garantizar el aseguramiento a las personas que tienen un contrato de trabajo, a los pensionados y a los trabajadores independientes con capacidad de pago; un régimen subsidiado para las personas más pobres y vulnerables de las áreas rural y urbana, sin capacidad de pago en el cubrimiento del monto total de la cotización establecida para acceder a los servicios; y un régimen de transición (vinculados) para las personas que no clasifiquen en ninguna de estas modalidades, dado que no cuentan con la capacidad de pago para hacer parte del contributivo y no son lo suficientemente pobres para estar en el subsidiado.

Con la creación del Régimen Subsidiado se reorienta la política de asignación de recursos en el sector público. El Sistema Nacional de Salud preexistente dirigía los recursos a los hospitales públicos (Oferta) para financiar la salud de los pobres lo cual resultó ineficiente. Ante tal problemática la Ley 60 de 1993 estableció criterios de asignación de recursos fiscales a los hospitales públicos de las rentas cedidas a los departamentos, pero bajo la modalidad de presupuesto prospectivo, no histórico, y se acabó la modalidad de transferencia para aparecer en contrato ínter-administrativo entre las direcciones seccionales y locales de salud y los hospitales de acuerdo a la cantidad de personas que atendieran (subsidio a la demanda).

En cuanto a la provisión de bienes públicos relacionados directamente con la salud se diseñó un plan de beneficios básico (Plan de Atención Básica -PAB) que cubre a todos los habitantes del territorio nacional de manera gratuita, abarca acciones de saneamiento ambiental, promoción de la salud, prevención de enfermedades, control de riesgos, vigilancia epidemiológica y atención de enfermedades de interés en salud pública. Este plan se financia con recursos de carácter fiscal y se ejecuta en el ámbito territorial a través de competencias establecidas para los departamentos, distritos y municipios.

El modelo anterior estaba centrado en la curación de la enfermedad, en la relación médico-paciente, la cual era impenetrable e innegociable, no se admitía ningún intermediario. Con la aplicación del principio de eficiencia, se requiere que los escasos recursos se utilicen de la mejor manera posible para que el beneficio alcance a la mayor cantidad de pobres existentes. Por tal motivación se introduce el concepto de medicina preventiva o de promoción en salud, concebida como la educación en salud para generar hábitos de vida saludable, cultura del auto-cuidado y la prevención de la enfermedad, tendiente a controlar los factores de riesgo condicionantes de la aparición de la enfermedad (externalidades positivas).

Las nuevas reglas del juego especifican que las responsabilidades del Estado deben enfocarse a dar garantía para que se presten servicios de salud de manera 
universal. De manera directa el Estado asumió tal responsabilidad al adecuar sus hospitales públicos en empresas descentralizadas y con autonomía técnica, financiera y administrativa (Empresas Sociales del Estado, ESE); de manera indirecta estructuró la prestación por medio de particulares (competencia regulada).

Este enfoque pretende integrar aquellos sub-sectores del antiguo esquema de salud en uno solo donde los principios regulatorios del Estado incentiven la competencia entre toda clase de organizaciones con el objetivo de brindar la mejor atención al usuario del servicio. Se propone en primera medida un esquema de Seguro universal regulado por el Estado que incluyera a todas las personas del territorio nacional, pero en vista de las diferencias económicas de los habitantes se crean dos tipos de aseguramiento: uno dirigido a aquellas personas vinculadas al mercado laboral y con capacidad de pago y otro dirigido hacia la población pobre y/o desempleada.

La integración, o mejor la Articulación, en palabras de Londoño (1997) se daría por medio de una nueva figura organizacional conocida como Empresas Promotoras de Servicios - EPS. Las cuales cumplen parte del papel de Patrocinador propuesto por Enthoven, se encargan de la afiliación, registro y recaudo de los afiliados al régimen contributivo, por delegación del Fondo de Seguridad y Garantía, FOSYGA. Deben cumplir normas específicas sobre condiciones de ingreso, seguridad y estabilidad de sus operaciones, cobertura y relaciones con sus usuarios además de cumplir otros requisitos de tipo administrativo y financiero exigidos por la superintendencia nacional de salud (Restrepo et al., 2004).

Figura 1. Funcionamiento del Sistema de salud colombiano bajo el modelo de competencia manejada

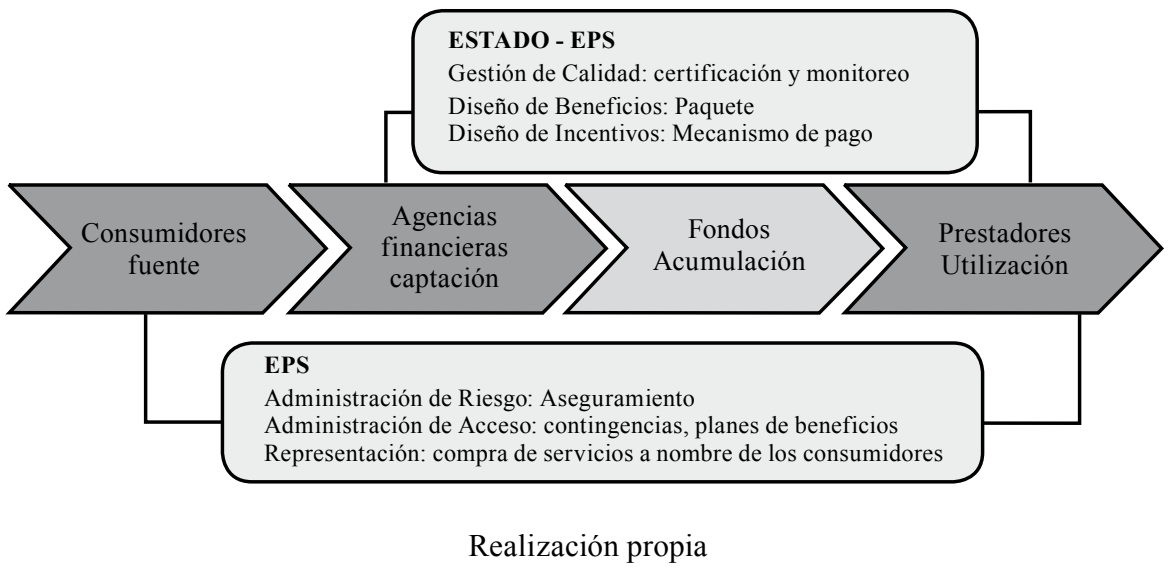


El Estado ejerce las funciones de regulación por medio de la concertación los principales actores del sistema en la figura del Consejo Nacional de Seguridad Social; la vigilancia y control del cumplimiento de las reglas definidas la realiza la Superintendencia; y el financiamiento de los subsidios la demanda y las acciones de salud pública le corresponden al Departamento de Nacional de Planeación y Entes territoriales por medio de las rentas cedidas.

En el modelo de la Ley 100 de 1993, los servicios individuales quedan organizados por la lógica de un mercado de aseguramiento con elementos de la teoría de Enthoven. Los mecanismos más importantes para el establecimiento de la función regulatoria en un ambiente competitivo son: la obligatoriedad del aseguramiento, la existencia de un fondo único al que confluyen todos los recursos (Fondo de Solidaridad y Garantía - FOSYGA), el pago de los aseguradores per cápita ajustado por riesgos (Unidad de Pago por Capitación -UPC) y la exigencia de un plan mínimo de beneficios (Plan Obligatorio de Salud - POS).

En este sistema, se separan las funciones de "administración del aseguramiento", a cargo de las Empresas Promotoras de Salud-EPS para el régimen contributivo y Administradoras de Régimen Subsidiado ${ }^{9}$ para el régimen de subsidios, de las de "prestación de servicios", en manos de las Instituciones Prestadoras de ServiciosIPS $^{10}$. Se esperaba que estas organizaciones confluyeran en un ambiente de competencia que garantizara alta calidad y proporcionaran información suficiente a los usuarios para que pudieran elegir, tanto su EPS (o ARS) como su IPS.

Se conservaron, hasta cierto momento, las instituciones más grandes que administraban los seguros obligatorios (Seguro Social, Caja Nacional de Previsión, entre otras), y se inició un proceso de autonomización de los hospitales públicos de servicios, con la denominación de Empresas Sociales del Estado (ESE). Se le obliga a estos hospitales a competir por los usuarios y establecer contratos con los administradores del aseguramiento (ARS y/o EPS). En general, el pago de las IPS por parte de las EPS se hace por facturación de servicios realizados, y las tarifas son definidas libremente por los aseguradores, con base en el comportamiento de la oferta y de la demanda.

Sin embargo, la evolución del sistema en más de una década evidencia que estos supuestos de competencia e información oportuna solo se aplican parcialmente en el régimen contributivo, puesto que el régimen subsidiado se ha caracterizado por alta burocratización e ineficiencia administrativa. Este problema se expresa en que a pesar de aumentar de manera progresiva el gasto en salud, las coberturas no cumplen sus objetivos iníciales de universalidad en el acceso a un paquete básico para el año 2001 .

9 Las ARS cumplen las mismas funciones que las EPS pero realizan contratos administrativos directamente con el Estado para administrar los recursos de subsidios a la demanda.

10 Las IPS son organizaciones de prestación de servicios (hospitales) tanto públicas como privadas. 
Tampoco se ha cumplido la meta de progresividad del plan de beneficios para el régimen subsidiado para alcanzar con igualdad los beneficios a que tienen derecho los afiliados al régimen contributivo. Lo cual crea un ambiente de inequidad en el acceso y la calidad en el sistema que lo hace continuar con las estructuras de prestación precarias anteriores.

Figura 2. Cobertura al SGSSS

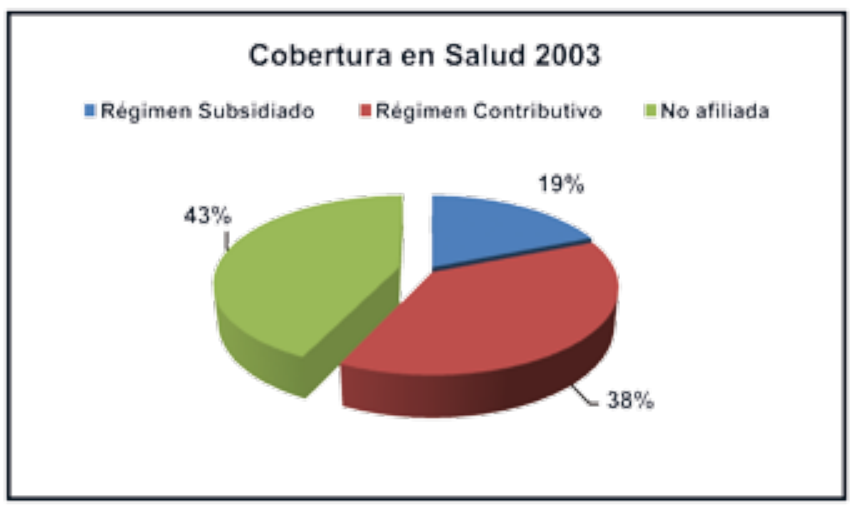

Fuente: Encuesta Calidad de Vida. DANE

Es totalmente cierto que existen grandes avances en el otorgamiento de subsidios a la demanda y en focalización (SISBEN), principalmente desde el año 2005 después de la instauración de subsidios parciales a la demanda, para aquellas personas que se encontraban por fuera del sistema en calidad de vinculados. Sin embargo, este esquema ha incentivado a las personas que carecen de recursos a competir por el subsidio debido a la falta de cobertura. Asimismo este ha sido utilizado infinitas veces para prácticas clientelistas y de corrupción. Lo cual es un obstáculo persistente en las funciones que ejerce el Estado en el sector.

También es supremamente rescatable el cubrimiento familiar que otorga el seguro y la conceptualización de la salud misma, así como el enfoque preventivo, pero si no existen condiciones de acceso y accesibilidad en igualdad al sistema de salud no se podrá avanzar para eliminar la fragmentación de los servicios y la marcada exclusión que se vive desde épocas de antaño. El problema es estructural no se puede limitar a una persona por su condición socioeconómica, definitivamente el Estado Social de Derecho en construcción debe velar por acabar estas barreras cada vez más poderosas para el desarrollo del país.

\section{CONCLUSIONES}

Los avances en la teoría económica has demostrado que tanto el Mercado como el Estado no son mecanismos eficientes para garantizar los servicios de salud a toda la 
población. Por un lado, el mercado en su forma libre es excluyente en la provisión pues sólo beneficia a aquellos con capacidad de adquirir los bienes y servicios. Además queda demostrado que el mercado no funciona tal como predice la teoría neoclásica en su esquema ideal, lo cual incentiva la intervención política. Por otro lado, el gobierno posee incentivos perversos que obstaculizan su funcionamiento óptimo en su papel interventor.

Particularmente en el caso colombiano el Estado ha demostrado ciertas falencias en su intervención en el sector salud lo cual ha llevado a revaluar los esquemas de regulación y transformarlos para responder a las necesidades de la población.

En la actualidad prevalecen esquemas que pretenden combinar las virtudes del Estado con las del mercado y así proveer bienes y servicios que mejoren el estado de la salud de las poblaciones. Este esquema está influenciado por la corriente neoinstitucionalista la cual parte de que los incentivos que otorgan reglas del juego bien definidas pueden solucionar las fallas de los mercados y establecer un óptimo desempeño en el sistema. Se busca competencia pero dirigida desde el Estado, hacia las actividades y sectores en las que sea conveniente para el bienestar general y la equidad.

En Colombia a pesar de las innovaciones que introdujo la reforma de la Ley 100 al sistema no se han logrado eliminar los problemas de fragmentación de los servicios persistentes desde hace un siglo, lo cual se evidencia con la persistente exclusión de gran parte de la población al acceso de los servicios. Aunque la ley diseñó mecanismos en pro de la articulación del sistema, ésta sólo se evidencia a nivel organizacional pues en la población ha institucionalizado su fragmentación según capacidad de pago.

La integración del sistema no se puede lograr si se diferencian las formas de prestación de servicios por grupos poblacionales lo cual atenta directamente contra el principio de equidad del que habla la Constitución, además es la principal barrera para conseguir la anhelada universalidad.

\section{REFERENCIAS}

Chicaiza, L. (200). El Mercado de la salud y la problemática del alto costo. Revista Problemas del desarrollo. Volumen 33, número 131, 2002. Disponible en: www.ejournal.unam.mx/pde/pde131/PDE13105.pdfwww.ejournal.unam.mx/pde/ pde131/PDE13105.pdf

Enthoven, A. (1993). The history and prin ciples of managed competition. Disponible en: http://content.healthaffairs.org/cgi/reprint/12/suppl_1/24

Hernández, M. (2002). Reforma sanitaria, equidad y derecho a la salud en Colombia. Cuadernos de Saúde Pública, vol. 18, no. 4, www.scielo.br

(1999). La fractura originaria en la formación del sistema de salud en Colombia: 1910-1946. Proceso sociopolítico y formulación de las reformas 
sanitarias en Colombia y Argentina 1910-1993 primera parte (mayo de 1999). 4-38. Observatorio en Políticas y Sistemas de salud. Universidad Nacional de Colombia. Disponible en:

http://www.revmed.unal.edu.co/politicas/original/Sistemas $\% 20 \mathrm{de} \% 20 \mathrm{salud} /$ tesismh1.pdf

Londoño, J., Frenk, J. (1997). Pluralismo Estructurado: Hacia Un Modelo Innovador para la Reforma de los Sistemas de Salud en América Latina. Banco Interamericano de Desarrollo. Documento de Trabajo 353. Disponible en:

www.iadb.org/res/publications/pubfiles/pubWP-353.pdf

North, D. (1993). Instituciones, Cambio Institucional y Desempeño Económico. Fondo de Cultura Económica.

OPS, 2002. La Organización Panamericana de la Salud Y El Estado Colombiano: Cien Años De Historia 1902 -2002.

Restrepo, J.; Rodríguez, S. (2002). Diseño y Experiencia de la Regulación en Salud en Colombia. Observatorio de la Salud, en: Revista de Economía Institucional. Primer semestre de 2005. Volumen 7. Universalidad de Externado de Colombia. Bogotá, Colombia.

Restrepo, M. (2000). La reforma de la seguridad en salud en Colombia y la teoría de la competencia regulada. Ensayos sobre el financiamiento de la seguridad social en salud. Los casos de Estados Unidos, Canadá, Argentina, Chile y Colombia. Vol. II. CEPAL.

Ministerio de Salud (1990). Reorganización del sistema nacional de salud. Una respuesta al cambio social. Memoria analítica de la ley 10 de 1990. Vol. I. Acomsap.

Sánchez, J. (1990). La Reforma al Sistema Nacional de Salud. Elementos teóricos para una interpretación estructural.

Orozco, J.M. (2006) Por qué reformar la reforma. Edición electrónica completo en: www.eumed.net/libros/2006/jmo/ Contenido.

Ortún, V. (1990). La economía en sanidad y medicina: instrumentos y limitaciones. Segunda Edición. Escola Universitaria de Treball Social. Editorial La Llar del Llibre.

Sen, Amartya (2002). ¿Por quéla equidad en salud?, en: Revista Panamericana Salud Pública, vol.11, no. 5. www.scielosp.org/scielo.php?script $=$ sci_arttext\&pid=S1020- 9 $892002000500005 \& \operatorname{lng}=$ en\&nrm=iso.

\section{Legislación:}

República de Colombia. Ley 715 de 2001. Sistema General de Participaciones. República de Colombia. Ley 100 de 1993.

República de Colombia. Ley 60 de 1993.

República de Colombia. Ley 10 de 1990. 